\title{
Lysophosphatidic acid increases the proliferation and migration of adipose-derived stem cells via the generation of reactive oxygen species
}

\author{
SANGJIN KANG ${ }^{1 *}$, JUHEE HAN ${ }^{1 *}$, SEUNG YONG SONG ${ }^{2}$, WON-SERK KIM ${ }^{3}$, SOYOUNG SHIN ${ }^{4}$, \\ JI HYE KIM ${ }^{5}$, HYOSUN AHN ${ }^{5}$, JIN-HYUN JEONG ${ }^{5}$, SUNG-JOO HWANG ${ }^{5}$ and JONG-HYUK SUNG ${ }^{5,6}$ \\ ${ }^{1}$ Department of Applied Bioscience, CHA University, Pocheon, Gyeonggi 487-010; \\ ${ }^{2}$ Department of Plastic and Reconstructive Surgery, Yonsei University College of Medicine, Seoul 120-749; \\ ${ }^{3}$ Department of Dermatology, Kangbuk Samsung Hospital, Sungkyunkwan University School of Medicine, Seoul 135-710; \\ ${ }^{4}$ Department of Pharmacy, College of Pharmacy, Wonkwang University, Iksan, Jeonbuk 570-749; ${ }^{5}$ Department of Pharmaceutics, \\ College of Pharmacy, Yonsei University, Incheon 406-840; ${ }^{6}$ Stemore Co. Ltd., Incheon 406-840, Republic of Korea
}

Received September 24, 2014; Accepted June 15, 2015

DOI: $10.3892 / \mathrm{mmr} .2015 .4023$

\begin{abstract}
Phospholipid derivatives, such as lysophosphatidic acid (LPA), exhibit mitogenic effects on mesenchymal stem cells; however, the molecular mechanism underlying this stimulation has yet to be identified. The aims of the present study were as follows: To evaluate the stimulatory effects of LPA on the proliferation and migration of adipose-derived stem cells (ASCs); to study the association between reactive oxygen species (ROS) and LPA signaling in ASCs; and to investigate the microRNAs upregulated by LPA treatment in ASCs. The results of the present study demonstrated that LPA increased the proliferation and migration of ASCs, and acted as a mitogenic signal via extracellular signal-regulated kinases $1 / 2$ and the phosphoinositide 3-kinase/Akt signaling pathways. The LPA1 receptor is highly expressed in ASCs, and pharmacological inhibition of it by Ki16425 significantly attenuated the proliferation and migration of ASCs. In addition, LPA treatment generated ROS via NADPH oxidase 4, and ROS were able to function as signaling molecules to increase the proliferation and migration of ASCs. The induction of ROS by LPA treatment also upregulated the expression of miR-210. A polymerase chain reaction array assay demonstrated that the expression levels of adrenomedullin and Serpine1 were increased following treatment with LPA. Furthermore, transfection with Serpinel-specific small interfering RNA
\end{abstract}

Correspondence to: Professor Jong-Hyuk Sung, Department of Pharmaceutics, College of Pharmacy, Yonsei University, 162-1 Songdo-dong, Incheon 406-840, Republic of Korea

E-mail: brian99@empal.com

${ }^{*}$ Contributed equally

Key words: adipose-derived stem cells, lysophosphatidic acid, reactive oxygen species, miR-210, Serpine1, mitogenic effects attenuated the migration of ASCs. In conclusion, the present study is the first, to the best of our knowledge, to report that ROS generation and miR-210 expression are associated with the LPA-induced stimulation of ASCs, and that Serpine1 mediates the LPA-induced migration of ASCs. These results further suggest that LPA may be used for ASC stimulation during stem cell expansion.

\section{Introduction}

Lysophosphatidic acid (LPA) is a phospholipid derivative that is synthesized by the removal of the choline group from lysophosphatidylcholine. LPA is an intermediate in the synthesis of phosphatidic acid and is able to act as a signaling molecule in various cell types (1-4). In particular, LPA acts as a potent mitogen via activation of high-affinity $G$ protein-coupled receptors, such as EDG2 and EDG4 $(5,6)$. Due to its ability to stimulate cell proliferation, aberrant LPA signaling has been linked to cancer progression, through numerous mechanisms. Dysregulation of the LPA receptors may lead to cellular hyperproliferation, which may in turn contribute to oncogenesis and metastasis (7-9). Signaling associated with LPA has also been reported to regulate the growth of fibroblasts, vascular smooth muscle cells, endothelial cells, and keratinocytes (10-13). In addition, the small GTPase Rho, a downstream molecule of the LPA receptor signal cascade, induces the formation of stress fibers and enhances cell migration (4). Therefore, LPA has been identified as a strong mitogenic factor. Recently, the molecular mechanisms underlying the mitogenic effects of LPA have been reported. Saunders et al (14) reported that LPA increases cell growth and inhibits apoptosis via the redox-dependent activation of extracellular signal-regulated kinases (ERK)-, Akt-, and nuclear factor- $\kappa \mathrm{B}$-dependent signaling pathways in ovarian cancer cells (14). Furthermore, the existence of an association between LPA signaling and reactive oxygen species (ROS)-mediated signaling has been suggested in other cell types (15-17). 
In addition to cancer cells, numerous studies have reported that LPA exerts stimulatory effects on mesenchymal stem cells (MSCs). LPA protects MSCs against hypoxia and serum deprivation-induced apoptosis via the ERK12 and phosphoinositide 3-kinase (PI3K)/Akt signaling pathways, and induces the migration of human lung-resident MSCs via the $\beta$-catenin pathway $(18,19)$. LPA also rescues MSCs from endoplasmic reticulum stress-associated apoptosis, induced by hypoxia and serum deprivation through the p38 pathway (20). In addition, LPA induces the osteoblastic differentiation of MSCs by increasing cyclic adenosine mono-phosphate and $\mathrm{Ca}^{2+}$ levels (21). LPA also affects the paracrine activity of MSCs, and stimulates secretion of vascular endothelial growth factor (VEGF) and stem cell-derived factor 1 (22). LPA promotes VEGF secretion by increasing the expression of $150 \mathrm{kDa}$ oxygen-related proteins in MSCs (23). However, there are few studies that specifically address the molecular mechanisms underlying LPA-induced ASC proliferation and migration. Our previous studies demonstrated that ROS generation exerts stimulatory effects on ASC proliferation and migration (24-27); however, the association between ROS and LPA signaling in ASCs remains poorly understood. Therefore, the present study aimed to investigate the stimulatory effects of LPA on ASC proliferation and migration, and the association between ROS and LPA signaling in ASCs, as well as the microRNA (miR) expression in ASCs following LPA treatment.

\section{Materials and methods}

Cell culture and chemical inhibition. The sampling of human subcutaneous adipose tissue, isolation of ASCs, and characterization of ASCs with ASC-specific surface markers using flow cytometry were performed as previously described $(28,29)$. Liposuction aspirates were obtained from a healthy female donor following the attainment of informed consent and approval from Bundang CHA Hospital (Seongnam, Korea; BD2011-152D). Human ASCs were cultured in $\alpha$-minimum essential medium (Gibco Life Technologies, Carlsbad, CA, USA) supplemented with $10 \%$ fetal bovine serum (Gibco Life Technologies) and 1\% penicillin-streptomycin (Gibco Life Technologies) at $37^{\circ} \mathrm{C}$ in a humidified atmosphere containing $5 \% \mathrm{CO}_{2}$.

For chemical inhibition studies, the ASCs were co-treated with 5 mM U0126 (ERK12 inhibitor; EMD Millipore, Billerica, MA, USA), 5 mM LY294002 (PI3KAkt inhibitor; EMD Millipore), 0.1-1 mM Ki16425 (Sigma-Aldrich, St. Louis, MO, USA) or oleoyl-L- $\alpha$-lysophosphatidic acid sodium salt (Sigma-Aldrich). For the inhibition of ROS generation, the ASCs were treated with $100 \mu \mathrm{M}$ NADPH oxidase (Nox) inhibitor diphenyleneiodonium chloride (DPI; Sigma-Aldrich).

Cell proliferation assay. ASCs were seeded at $7 \times 10^{3}$ cells/well in 48-well plates. Following incubation with the LPA (1-50 $\mu \mathrm{M})$ for 48 or $72 \mathrm{~h}$, cell numbers were measured using the Cell Counting kit (CCK)-8 Assay (Dojindo Molecular Technologies, Inc., Rockville, MD, USA). Briefly, the medium in each well was replaced with medium containing the water-soluble tetrazolium salt WST-8 (10\% vv). Following incubation for $2 \mathrm{~h}$ at $37^{\circ} \mathrm{C}$, absorbance was measured at $450 \mathrm{~nm}$ using a Sunrise ${ }^{\mathrm{TM}}$ Microplate Absorbance Reader (Tecan Group Ltd., Männedorf, Switzerland).

Cell migration assay. Cell migration was measured using a transwell chamber. The transwell inserts with an $8 \mu \mathrm{m}$ 3422 pore polycarbonate membrane (Corning Life Sciences, Cambridge, MA, USA), were coated with $0.1 \%$ gelatin (Sigma-Aldrich) in phosphate-buffered saline (PBS) for $2 \mathrm{~h}$ at $37^{\circ} \mathrm{C}$. The ASCs were seeded at $1 \times 10^{4}$ cells per $200 \mu 1$ in the upper chamber and incubated for $2 \mathrm{~h}$ at $37^{\circ} \mathrm{C}$ in an atmosphere containing $5 \% \mathrm{CO}_{2}$. The ASCs were allowed to migrate towards $500 \mu \mathrm{l}$ medium containing $10 \mu \mathrm{M}$ LPA in the lower chamber. Migration induced by serum-free medium was used as a negative control. Following $16 \mathrm{~h}$ incubation at $37^{\circ} \mathrm{C}$, the transwell inserts were fixed with ice-cold $100 \%$ methanol for $20 \mathrm{~min}$ and stained with $0.23 \%$ crystal violet (Sigma-Aldrich) in $2 \%$ ethanol for 30 min. Following further washing with distilled water, the non-migratory cells were removed by gently wiping the upper face of the transwell inserts with a cotton swab. Images of the stained cells were captured using a light microscope (CKX41; Olympus, Tokyo, Japan) and were analyzed using Image J 1.47 software (National Institutes of Health, Bethesda, MD, USA), in order to determine the number of cells that had migrated to the lower side of membrane.

Intracellular ROS staining. Intracellular ROS production was measured by flow cytometry using the ROS-sensitive dye 2',7'-dichlorofluorescin diacetate (DCF-DA; Molecular Probes Life Technologies, Carlsbad, CA, USA), as previously described $(25,26,30)$. Briefly, the ASCs were seeded at $2.5 \times 10^{5}$ cells $/ 60 \mathrm{~mm}$ dish. The following day, the cells were incubated with $20 \mu \mathrm{M}$ DCF-DA for $10 \mathrm{~min}$ at $37^{\circ} \mathrm{C}$, and treated with LPA for $20 \mathrm{~min}$ at $37^{\circ} \mathrm{C}$. Following incubation, the cells were trypsinized and resuspended in PBS. Green fluorescence intensity was measured using a BD FACSCalibur flow cytometer and analyzed using CellQuest Pro software (BD Biosciences, Franklin Lakes, NJ, USA).

Polymerase chain reaction (PCR) array and reverse transcription-quantitative PCR $(R T-q P C R)$. Total RNA was isolated using the RNeasy kit (Qiagen, Inc., Valencia, CA, USA), and was reverse transcribed using a cDNA Synthesis kit (Promega Corporation, Madison, WI, USA), according to the manufacturer's instructions.

The synthesized cDNA was used in a PCR Array analysis using a CFX96 Real-Time PCR Detection system (Bio-Rad Laboratories, Inc., Hercules, CA, USA), according to the manufacturer's instructions. A PAHS-014ZD Human Signal Transduction Pathway Finder RT2 Profiler PCR Array (Qiagen, Inc.), which was composed of 84 transcripts representing 10 distinct signaling pathways, was used to identify the genes associated with signaling pathways that may be affected by LPA treatment. Differential gene expression and statistical analysis of the data were performed using PCR Array Data Analysis software (Qiagen, Inc.).

RT-qPCR was performed using a StepOne Plus Real-Time PCR system (Applied Biosystems Life Technologies, Foster City, CA, USA) using the following primers: Adrenomedullin 
(ADM) forward, CTTATTCGGCCCCAGGACAT, and reverse, ACTGGTAGATCTGGTGTGCC; Serpinel forward, CCGCCTCTTCCACAAATCAG, and reverse, AATGTT GGTGAGGGCAGAGA; LPA receptor 1 (LPAR1) forward, TCATCTGGACTATGGCCATC, and reverse, CAAGTT GAAAATGGCCCAGA; LPAR2 forward, CAATGCTGC TGTGTACTCTT, and reverse, TGGGCAGAGGATGTA TAGTG; LPAR3 forward, CTACAAGGACGAGGACATGT, and reverse, ATCCTCTATGTACTGGCTGC; LPAR4 forward, TCTGCAAGATCTCTGGAACT, and reverse, ACA CAATGGCAGAATTCCTC; LPAR5 forward, TTCTCC CGTGTCCTGACTA, and reverse, ACATGTACACGCTCA CCAC; LPAR6 forward, TTGTATGGGTGCATGTTCAG, and reverse, CAAGTCTGACATTGCCAAGT; and GAPDH forward, CGAGATCCCTCCAAAATCAA, and reverse, TGT GGTCATGAGTCCTTCCA. GAPDH served as a loading control. Thermal cycling over 40 cycles consisted of an initial denaturation at $95^{\circ} \mathrm{C}$ for $10 \mathrm{~min}$, then $95^{\circ} \mathrm{C}$ for $15 \mathrm{sec}, 60^{\circ} \mathrm{C}$ for $1 \mathrm{~min}$ and $95^{\circ} \mathrm{C}$ for $15 \mathrm{sec}$, and was terminated by a final extension at $60^{\circ} \mathrm{C}$ for $1 \mathrm{~min}$.

Measurement of the expression levels of miR-210. Total RNA was extracted using TRIzol ${ }^{\circledR}$ reagent (Invitrogen Life Technologies, Carlsbad, CA, USA), and $0.5 \mu \mathrm{g}$ total RNA was reverse transcribed using a Mir-X miRNA First-Strand Synthesis kit (Clontech Laboratories, Inc., Mountain View, CA, USA) according to the manufacturer's instructions. The expression levels of miR-210 were measured by RT-qPCR using the miR-210 primer (CTGTGCGTGTGACAGCGG CTGA; Genolution Pharmaceuticals, Inc., Seoul, Korea) as previously described (24). U6 small nuclear RNA served as a loading control (Clontech Laboratories, Inc.).

Transfection with small interfering (si)RNA and miR-210 inhibitor. The Stealth RNAi ${ }^{\mathrm{TM}}$ siRNA specific to human Nox4 (sense, UUAUCCAACAAUCUCCUGGUUCUCC; and antisense, GGAGAACCAGGAGAUUGUUGGAUAA), the Silencer Select siRNA for human Serpinel (ID\# s10013), and the non-targeting negative control (scramble) siRNA (Silencer Select Negative Control\#1 siRNA) were purchased from Invitrogen Life Technologies. The miR-210 inhibitor (hsa-miR210) and the miRNA inhibitor negative control were purchased from Genolution Pharmaceuticals, Inc. ASCs were seeded in $60 \mathrm{~mm}$ dishes at a density of $2 \times 10^{6}$ with complete medium. The following day, confluent ASCs were maintained in serum-free medium without antibiotics for $2 \mathrm{~h}$. Subsequently, the ASCs were transfected with $20 \mathrm{nM}$ of each siRNA or miR-210 inhibitor using Lipofectamine ${ }^{\circledR} 2000$ transfection reagent (Invitrogen Life Technologies), according to the manufacturer's instructions in 24-well plates or $60 \mathrm{~mm}$ dishes.

Western blotting. Proteins of ASCs were isolated using SDS sample buffer. The soluble protein concentration was determined using the Quick start Bradford $1 \mathrm{X}$ dye reagent (Bio-Rad Laboratories, Inc.). The western blot analysis was performed as previously described (25) using antibodies targeting p-ERK12 (1:2,000; cat. no. 9106; in 5\% milk), ERK12 (1:4,000; cat. no. 9102; in 5\% milk), p-Akt (1:1,000; cat. no. 9271; in 5\% milk), and Akt (1:4,000; cat. no. 9272; in
Table I. LPAR expression in adipose-derived stem cells.

\begin{tabular}{lc}
\hline Gene & Ct-value \\
\hline LPAR1 & $24.44 \pm 0.50$ \\
LPAR2 & $29.82 \pm 0.78$ \\
LPAR3 & $30.85 \pm 0.94$ \\
LPAR4 & $30.84 \pm 0.90$ \\
LPAR5 & $31.44 \pm 0.54$ \\
LPAR6 & $29.60 \pm 0.57$ \\
GAPDH & $18.84 \pm 0.09$
\end{tabular}

Ct, cycle threshold; LPAR, lysophophatidic acid receptor.

5\% milk). All antibodies were purchased from Cell Signaling Technology, Inc. (Danvers, MA, USA). Horseradishperoxidase (HRP)-conjugated secondary mouse antibody $(1: 10,000)$ was purchased from Vector Laboratories, Inc. (Burlingame, CA, USA) and HRP-conjugated secondary rabbit antibody was purchased from Cell Signaling Technology, Inc. (1:10,000; cat. no. 7074).

Statistical analysis. All data are presented as the mean \pm standard deviation. Statistical analysis of the data was performed using Microsoft Excel software (Microsoft Corporation, Redmond, WA, USA). A Student's t-test was used to analyze the data. $\mathrm{P}<0.05$ was considered to indicate a statistically significant difference.

\section{Results}

LPA increases the proliferation and migration of ASCs. The present study initially investigated whether LPA treatment increased the proliferation and migration of ASCs. As expected, 1-50 mM LPA significantly increased the proliferation (Fig. 1A) and migration (Fig. 1B) of ASCs in a dose-dependent manner. Since PI3K/Akt and mitogen-activated protein kinase (MAPK) signaling pathways have previously been reported to be associated with the mitogenic effects of LPA, the phosphorylation levels of Akt and ERK12 were measured following LPA treatment, and phosphorylation of both proteins increased in a time-dependent manner (Fig. 1C). In the inhibition study, pharmacological inhibition of PI3K/Akt by LY294002 significantly reduced the LPA-induced proliferation of ASCs (Fig. 1D). In addition, pharmacological inhibition of PI3K/Akt by LY294002, and of ERK by U0126 significantly reduced the LPA-induced migration of ASCs (Fig. 1E). These results suggest that LPA acts as a strong mitogenic factor in ASCs.

LPA mediates the mitogenic effects of ASCs via the LPA receptor. The expression levels of LPAR were measured in the ASCs (Table I), indicating that LPAR1 is highly expressed in ASCs, as compared with the other isoforms. To measure the involvement of LPAR in the mitogenic function of LPA, pharmacological inhibition of LPAR by Ki16425 was performed. Co-treatment with Ki16425 (0.1 and $1 \mathrm{mM})$ significantly 
A

B

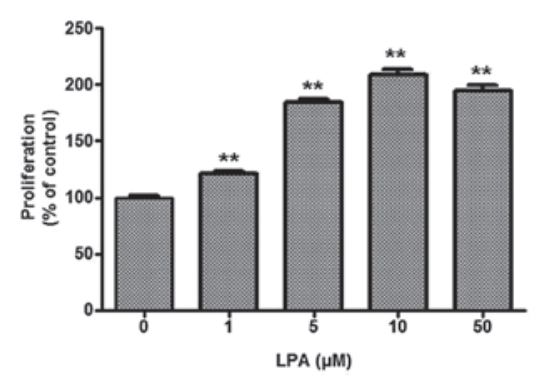

Control
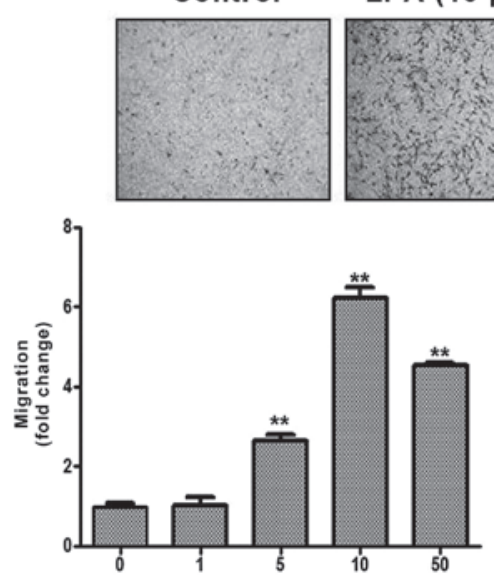

$\mathbf{E}$
LPA $(10 \mu \mathrm{M})$

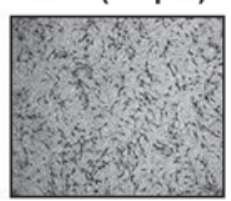

C

D

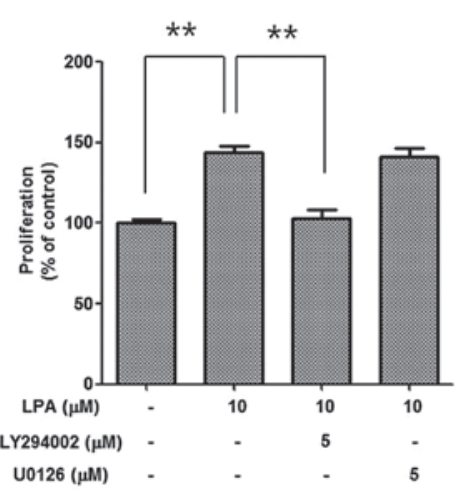

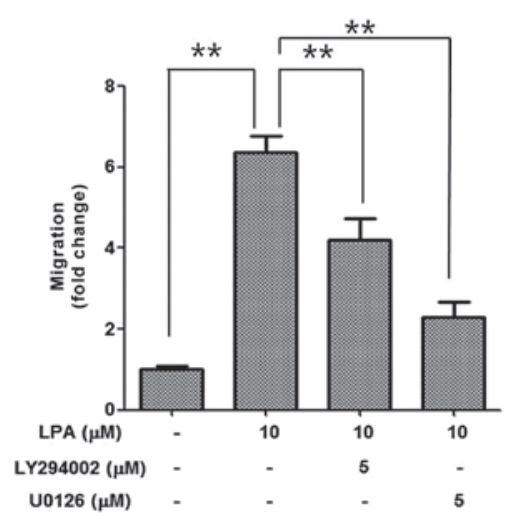

Figure 1. Lysophosphatidic acid (LPA) increases the proliferation and migration of adipose-derived stem cells (ASCs). (A and B) LPA (1-50 mM) significantly increased the (A) proliferation and (B) migration of ASCs, as measured by the Cell Counting kit (CCK)-8 Assay and transwell chambers, respectively. (C) Western blot analysis of the phosphorylation of Akt and extracellular signal-regulated kinases (ERK)1/2 showed that band density increased following LPA treatment. (D) Pharmacological inhibition of phophoinositide 3-kinase (PI3K)/Akt by LY294002 significantly reduced the LPA-induced proliferation of ASCs. (E) Pharmacologic inhibition of PI3K/Akt by LY294002 and of ERK by U0126 significantly reduced the LPA-induced migration of ASCs. Data are presented as the mean \pm standard deviation. ${ }^{*} \mathrm{P}<0.05$, and ${ }^{* *} \mathrm{P}<0.01$.

A

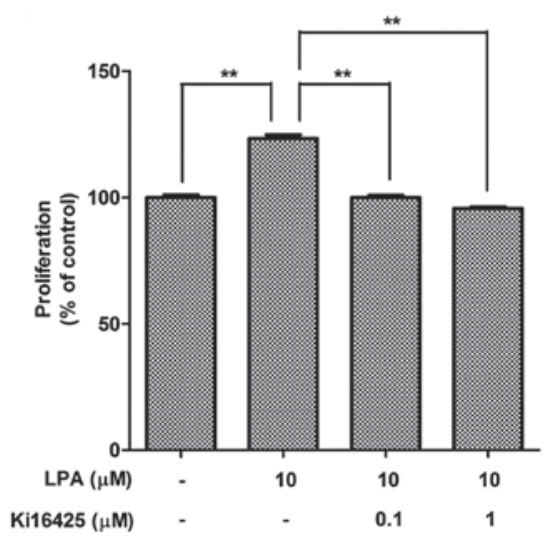

B

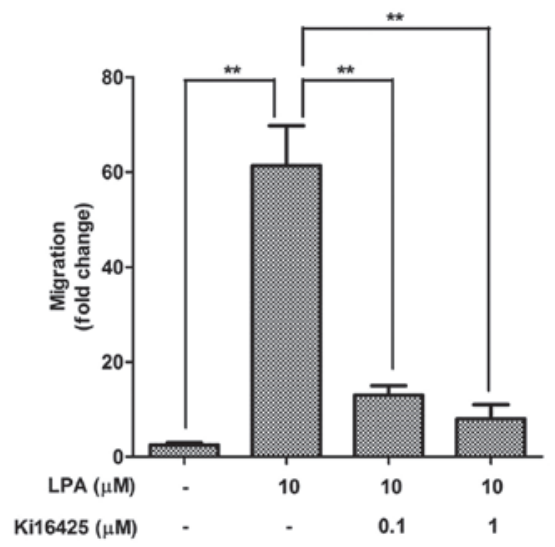

Figure 2. Lysophosphatidic acid (LPA) mediates the mitogenic effects of adipose-derived stem cells (ASCs) via LPA receptors (LPAR). Pharmacological inhibition of LPAR by Ki16425 (0.1 and $1 \mathrm{mM}$ ) significantly attenuated the LPA-induced (A) proliferation and (B) migration of ASCs. Data are presented as the mean \pm standard deviation. ${ }^{* *} \mathrm{P}<0.01$.

attenuated LPA-induced proliferation (Fig. 2A) and migration (Fig. 2B) of ASCs.
LPA treatment induces ROS generation. The present study also investigated whether the LPA-induced proliferation 
A

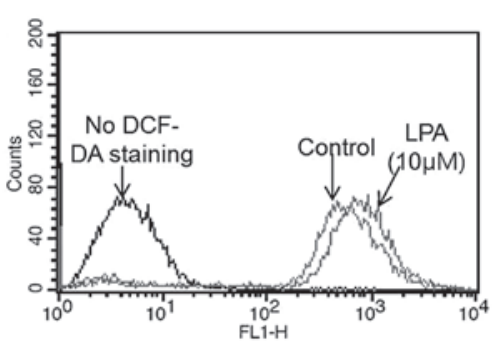

C

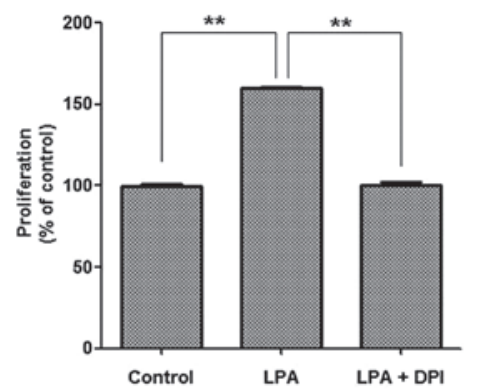

$\mathbf{E}$

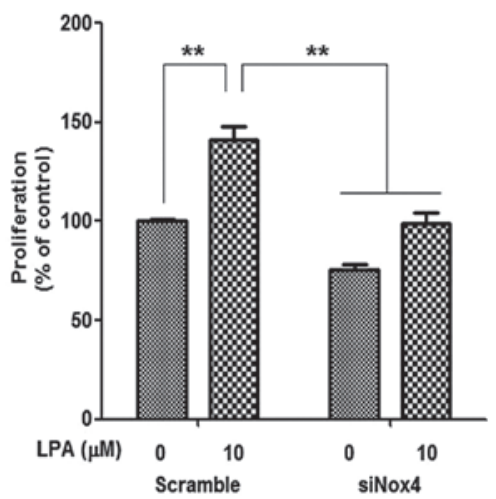

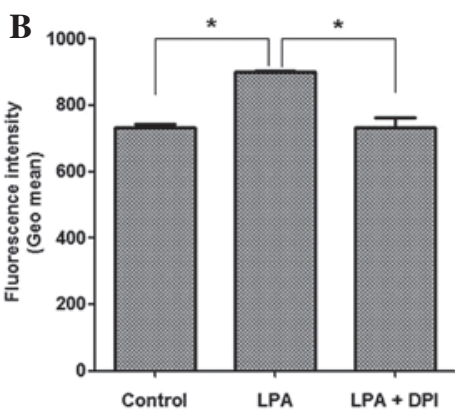

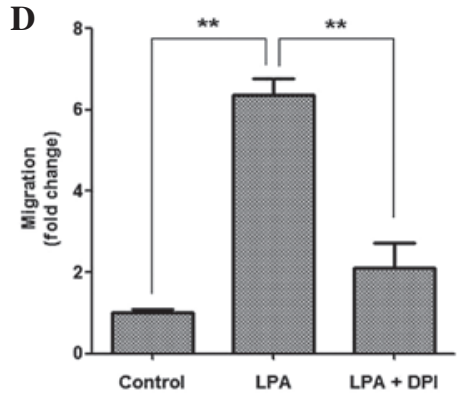

F

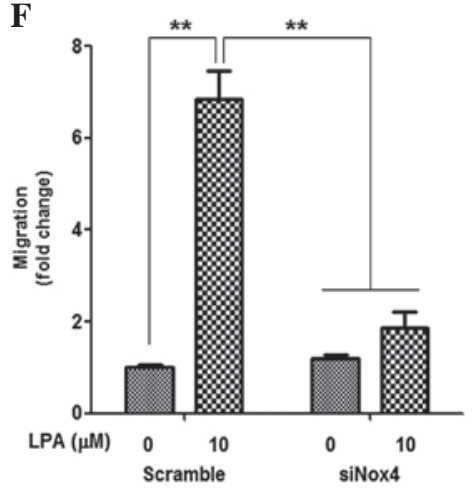

Figure 3. Lysophosphatidic acid (LPA) treatment induces reactive oxygen species generation. (A) LPA treatment significantly increased the fluorescence intensity of dichlorofluorescin diacetate (DCF-DA) assessed by flow cytometry. (B) Pharmacological inhibition of NADPH oxidase (Nox) by diphenyleneiodonium (DPI) significantly reduced the fluorescence intensity of DCF-DA. (C and D) DPI treatment significantly reduced the LPA-induced (C) proliferation and (D) migration of ASCs. (E and F) Downregulation of Nox4 by small interfering (si)RNA transfection significantly decreased the LPA-induced (E) proliferation and (F) migration of ASCs. Data are presented as the mean \pm standard deviation. ${ }^{*} \mathrm{P}<0.05,{ }^{* *} \mathrm{P}<0.01$.

and migration of ASCs was mediated by ROS generation. As expected, LPA treatment significantly increased the fluorescence intensity of DCF-DA, as determined by flow cytometric analysis (Fig. 3A); and pharmacological inhibition of Nox following treatment with DPI significantly attenuated the fluorescence intensity of DCF-DA (Fig. 3B). In addition, DPI treatment significantly reduced LPA-induced proliferation (Fig. 3C) and migration (Fig. 3D) of ASCs. As Nox4 is highly expressed in ASCs and primarily mediates ROS generation (26), downregulation of Nox4 expression in ASCs by siRNA transfection and Nox4 silencing significantly decreased LPA-induced cell proliferation (Fig. 3E) and migration (Fig. 3F). These results suggest that Nox4 may be associated with LPA-mediated ROS generation and downstream mitogenic effects in ASCs.

LPA increases miR-210 expression. Our previous study demonstrated that ROS generation induced by hypoxia, or chemicals, induced miR-210 expression and increased the proliferation and migration of ASCs (24); therefore, the present study investigated the involvement of miR-210 in the LPA-induced proliferation and migration of ASCs. As expected, LPA treatment increased miR-210 expression (Fig. 4A); however, the extent of upregulation was not as great as that induced by hypoxia and chemical ROS donors. In the inhibition study, miR-210 inhibitors significantly attenuated LPA-induced proliferation (Fig. 4B) and migration (Fig. 4C) of ASCs. These results suggest that miR-210 is involved in the LPA-induced proliferation and migration of ASCs.

LPA upregulates the expression of ADM and Serpinel. The human Signal Transduction Pathway Finder RT2 Profiler PCR Array was used to identify the LPA target genes that mediated the proliferation and migration of ASCs. A total of $4 \mathrm{~h}$ following LPA treatment, the expression levels of ADM increased 5.12-fold, as demonstrated by the PCR array (Fig. 5A). RT-qPCR analysis confirmed that the expression levels of ADM were upregulated $\sim 6$-fold (Fig. 5B). A total of $24 \mathrm{~h}$ following LPA treatment, the expression levels of Serpine1 increased 8.28-fold, as determined by the PCR array (Fig. 5C), and $\sim 18$-fold, as determined by the RT-qPCR (Fig. 5D). Therefore, the present study investigated whether ADM 
A

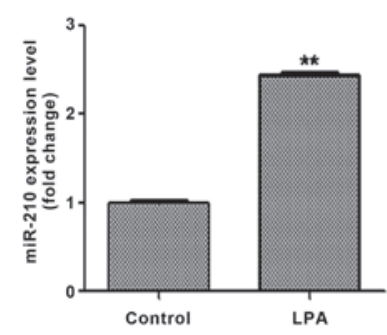

B

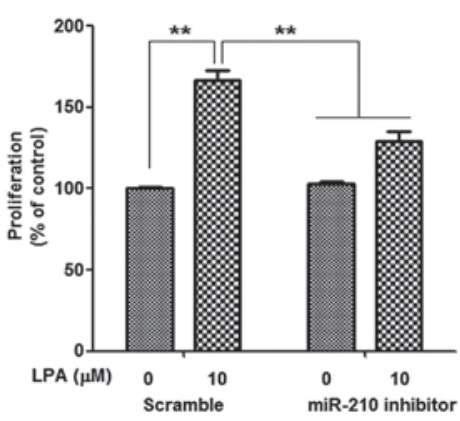

C

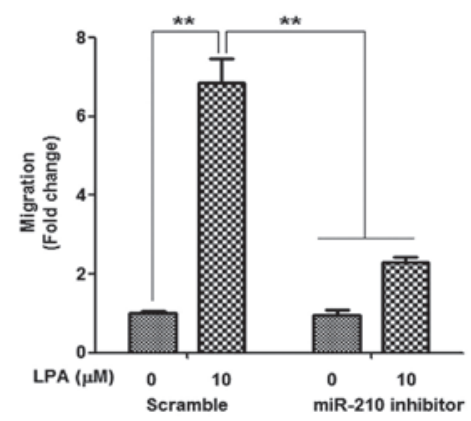

Figure 4. Lysophosphatidic acid (LPA) increases the expression levels of microRNA (miR)-210. (A) Reverse transcription-quantitative polymerase chain reaction analysis demonstrated that LPA treatment increased the expression levels of miR-210 in the adipose-derived stem cells (ASCs). Transfection with miR-210 inhibitor significantly attenuated the LPA-induced (B) proliferation and $(\mathrm{C})$ migration of ASCs. Data are presented as the mean \pm standard deviation. ${ }^{* *} \mathrm{P}<0.01$.

A

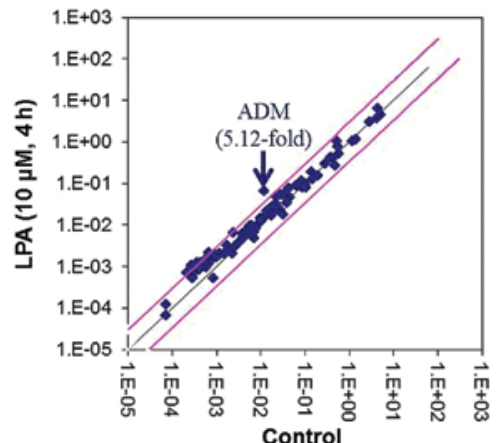

C

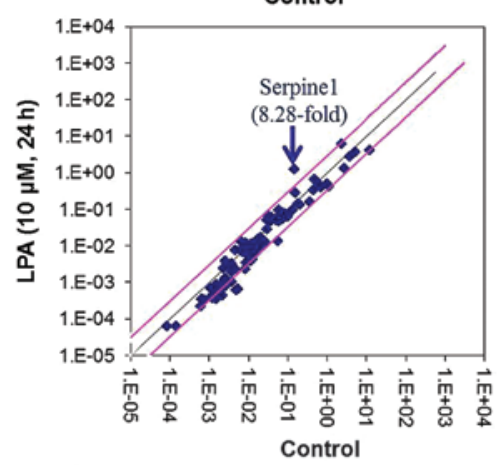

B

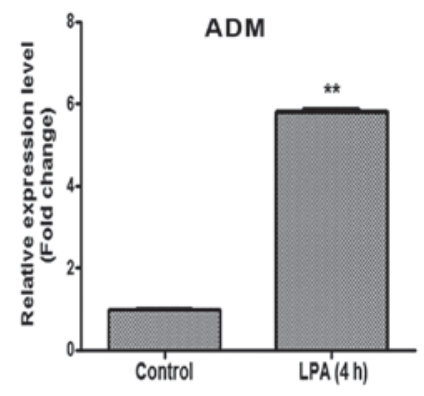

D

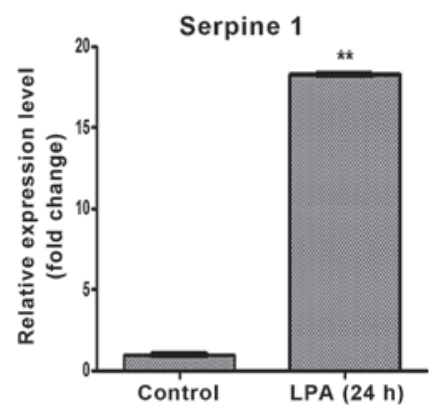

$\mathbf{E}$

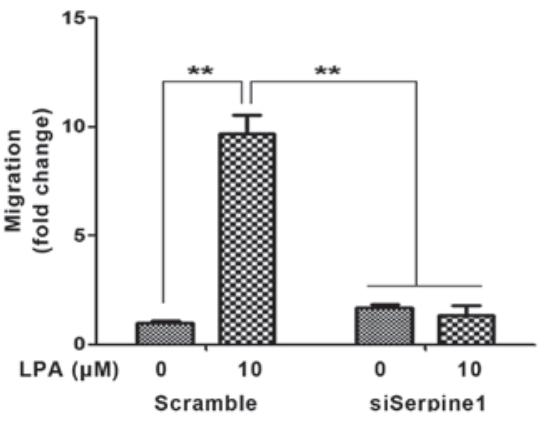

Figure 5. Lysophosphatidic acid (LPA) upregulates the expression levels of adrenomedullin (ADM) and Serpine1, and mediates the migration of adipose-derived stem cells (ASCs) via Serpine1. (A) Identification of the target genes of LPA that mediated the proliferation and migration of ASCs using a Human Signal Transduction Pathway Finder RT2 Profiler Polymerase Chain Reaction (PCR) Array. The expression levels of ADM significantly increased $4 \mathrm{~h}$ following LPA treatment. (B) Confirmation of the upregulation of ADM expression was provided by reverse transcription quantitative RT- $\mathrm{q}(\mathrm{PCR})$ analysis. (C) The expression levels of Serpinel increased $24 \mathrm{~h}$ following LPA treatment, as determined by PCR array. (D) Increased Serpine1 expression as determined by RT-qPCR. (E) Transfection with Serpine1 small interfering (si)RNA significantly attenuated LPA-induced migration of ASCs. The pink lines indicate a 3-fold change in gene expression between the control and LPA treatment. Data are presented as the mean \pm standard deviation. ${ }^{* *} \mathrm{P}<0.01$.

and/or Serpine1 mediated LPA-induced proliferation and migration of ASCs using siRNAs specific for ADM and Serpine1. Although silencing of ADM did not affect proliferation or migration (data not shown), transfection with Serpine1 siRNA significantly attenuated the LPA-induced migration of ASCs (Fig. 5E). 


\section{Discussion}

The present study investigated the signal pathways and molecular mechanisms underlying LPA-induced proliferation and migration of ASCs. Previous studies have demonstrated that LPA is able to mediate the proliferation and migration of various cells, primarily via the ERK12 and PI3K/Akt signaling pathways $(19,31,32)$. The results of the present study demonstrated that LPA may function as a mitogenic signal via LPARs, and the ERK12 and PI3K/Akt signaling pathways in ASCs. In addition to these signaling pathways, the present study demonstrated that LPA treatment induced ROS generation via Nox4, and that these ROS act as signaling molecules to increase the proliferation and migration of ASCs. Furthermore, upregulation of miR-210 expression by ROS may contribute to the increased proliferation and migration of ASCs, results which are concordant with those of a previous study (24). LPA also upregulated Serpinel expression, which increased ASC migration.

As determined by PCR array, the expression levels of ADM and Serpinel increased 4 and $24 \mathrm{~h}$ following LPA treatment, respectively. In addition, the results of the present study demonstrated that Serpine1 mediated LPA-induced migration of ASCs; however, no proliferation-associated genes were identified in the present study. Although the involvement of ADM in ASC proliferation was hypothesized, siRNA silencing of ADM did not inhibit the proliferation of ASCs. A previous study also reported that ADM did not increase, but rather inhibited, LPA-induced proliferation, and attenuated the stimulation of the MAPK pathway by LPA treatment in adventitial cells (33). Therefore, the mediator(s) of LPA-induced proliferation of ASCs have yet to be identified.

miRNAs exert their actions primarily at the post-transcriptional level, and their expression is known to be regulated by redox signaling (24,34-36). In our previous study, the expression levels of miR-210 were significantly increased via various ROS generators (hypoxia, antimycin, rotenone, and platelet-derived growth factor subunit B), and regulated the proliferation and migration of ASCs (24). The present study demonstrated that LPA-induced ROS generation upregulated miR-210 expression in ASCs, and increased the proliferation and migration of ASCs. However, the extent of miR-210 LPA-induced upregulation was lower, as compared with other ROS generators such as hypoxia. In addition, ROS generation by S1P did not induce miR-210 expression in ASCs (data not shown). Therefore, it is reasonable to assume that ROS generation is not a prerequisite for the upregulation of miR-210 expression in ASCs.

In conclusion, the present study demonstrated that LPA increases the proliferation and migration of ASCs via Nox4-induced ROS generation. The present study is the first, to the best of our knowledge, to demonstrate that miR-210 expression is associated with LPA-induced stimulation, and that Serpine1 mediates the LPA-induced migration of ASCs. Therefore, phospholipid derivatives, such as LPA and S1P, may be used to stimulate ASCs during stem cell expansion.

\section{Acknowledgements}

The present study was supported by a grant from the Yonsei University Research Fund (grant no. 2014-12-0040).

\section{References}

1. Glazier AT, Blackmore PF, Nolan RD and Wasilenko WJ: Attenuation of LPA-mediated calcium signaling and inositol polyphosphate production in rat-1 fibroblasts transformed by the v-src oncogene. Biochem Biophys Res Commun 245: 607-612, 1998.

2. Jonkers J and Moolenaar WH: Mammary tumorigenesis through LPA receptor signaling. Cancer Cell 15: 457-459, 2009.

3. Sorensen SD, Nicole O, Peavy RD, Montoya LM, Lee CJ, Murphy TJ, Traynelis SF and Hepler JR: Common signaling pathways link activation of murine PAR-1,LPA, and S1P receptors to proliferation of astrocytes. Mol Pharmacol 64: 1199-1209, 2003.

4. Tangkijvanich P, Melton AC, Santiskulvong C and Yee HF Jr: Rho and p38 MAP kinase signaling pathways mediate LPA-stimulated hepatic myofibroblast migration. J Biomed Sci 10: 352-358, 2003.

5. Bandoh K, Aoki J, Taira A, Tsujimoto M, Arai H and Inoue K: Lysophosphatidic acid (LPA) receptors of the EDG family are differentially activated by LPA species. Structure-activity relationship of cloned LPA receptors. FEBS Lett 478: 159-165, 2000.

6. Yanagida K, Kurikawa Y, Shimizu T and Ishii S: Current progress in non-Edg family LPA receptor research. Biochim Biophys Acta 1831: 33-41, 2013.

7. Hao F, Tan M, Xu X, Han J, Miller DD, Tigyi G and Cui MZ: Lysophosphatidic acid induces prostate cancer PC3 cell migration via activation of LPA(1), p42 and p38alpha. Biochim Biophys Acta 1771: 883-892, 2007.

8. Sengupta S, Xiao YJ and Xu Y: A novel laminin-induced LPA autocrine loop in the migration of ovarian cancer cells. FASEB J 17: 1570-1572, 2003.

9. Shida D, Watanabe T, Aoki J, Hama K, Kitayama J, Sonoda H, Kishi Y, Yamaguchi H, Sasaki S, Sako A, et al: Aberrant expression of lysophosphatidic acid (LPA) receptors in human colorectal cancer. Lab Invest 84: 1352-1362, 2004.

10. Kwon YJ, Sun Y, Kim NH and Huh SO: Phosphorylation of CREB, a cyclic AMP responsive element binding protein, contributes partially to lysophosphatidic acid-induced fibroblast cell proliferation. Biochem Biophys Res Commun 380: 655-659, 2009.

11. Lee H, Goetzl EJ and An S: Lysophosphatidic acid and sphingosine 1-phosphate stimulate endothelial cell wound healing. Am J Physiol Cell Physiol 278: C612-C618, 2000.

12. Sauer B, Vogler R, Zimmermann K, Fujii M, Anzano MB, Schäfer-Korting M, Roberts AB and Kleuser B: Lysophosphatidic acid interacts with transforming growth factor-beta signaling to mediate keratinocyte growth arrest and chemotaxis. J Invest Dermatol 123: 840-849, 2004.

13. Tokumura A, Iimori M, Nishioka Y, Kitahara M, Sakashita M and Tanaka S: Lysophosphatidic acids induce proliferation of cultured vascular smooth muscle cells from rat aorta. Am J Physiol 267: C204-C210, 1994.

14. Saunders JA, Rogers LC, Klomsiri C, Poole LB and Daniel LW: Reactive oxygen species mediate lysophosphatidic acid induced signaling in ovarian cancer cells. Free Radic Biol Med 49: 2058-2067, 2010.

15. Chen Q, Olashaw $\mathrm{N}$ and $\mathrm{Wu} \mathrm{J}$ : Participation of reactive oxygen species in the lysophosphatidic acid-stimulated mitogen-activated protein kinase kinase activation pathway. $\mathrm{J}$ Biol Chem 270: 28499-28502, 1995.

16. Idzko M, Laut M, Panther E, Sorichter S, Dürk T, Fluhr JW, Herouy Y, Mockenhaupt M, Myrtek D, Elsner P, and Norgauer J: Lysophosphatidic acid induces chemotaxis, oxygen radical production, $\mathrm{CD} 11 \mathrm{~b}$ up-regulation, $\mathrm{Ca}^{2+}$ mobilization, and actin reorganization in human eosinophils via pertussis toxin-sensitive G proteins. J Immunol 172: 4480-4485, 2004.

17. Lin CC, Lin CE, Lin YC, Ju TK, Huang YL, Lee MS, Chen JH and Lee H: Lysophosphatidic acid induces reactive oxygen species generation by activating protein kinase $\mathrm{C}$ in $\mathrm{PC}-3$ human prostate cancer cells. Biochem Biophys Res Commun 440: 564-569, 2013.

18. Badri L and Lama VN: Lysophosphatidic acid induces migration of human lung-resident mesenchymal stem cells through the $\beta$-catenin pathway. Stem Cells 30: 2010-2019, 2012.

19. Chen J, Baydoun AR, Xu R, Deng L, Liu X, Zhu W, Shi L, Cong X, $\mathrm{Hu} \mathrm{S}$ and Chen X: Lysophosphatidic acid protects mesenchymal stem cells against hypoxia and serum deprivation-induced apoptosis. Stem Cells 26: 135-145, 2008.

20. Li Z, Wei H, Liu X, Hu S, Cong X and Chen X: LPA rescues ER stress-associated apoptosis in hypoxia and serum deprivation-stimulated mesenchymal stem cells. J Cell Biochem 111: 811-820, 2010. 
21. Liu YB, Kharode Y, Bodine PV, Yaworsky PJ, Robinson JA and Billiard J: LPA induces osteoblast differentiation through interplay of two receptors: LPA1 and LPA4. J Cell Biochem 109: 794-800, 2010.

22. Jeon ES, Heo SC, Lee IH, Choi YJ, Park JH, Choi KU, Park do Y, Suh DS, Yoon MS and Kim JH: Ovarian cancer-derived lysophosphatidic acid stimulates secretion of VEGF and stroma cell-derived factor-1 alpha from human mesenchymal stem cells. Exp Mol Med 42: 280-293, 2010.

23. Wei H, Wang F, Wang X, Yang J, Li Z, Cong X and Chen X: Lysophosphatidic acid promotes secretion of VEGF by increasing expression of $150-\mathrm{kD}$ Oxygen-regulated protein (ORP150) in mesenchymal stem cells. Biochim Biophys Acta 1831: 1426-1434, 2013.

24. Kim JH, Park SG, Song SY, Kim JK and Sung JH: Reactive oxygen species-responsive miR-210 regulates proliferation and migration of adipose-derived stem cells via PTPN2. Cell Death Dis 4: e588, 2013.

25. Kim JH, Park SH, Park SG, Choi JS, Xia Y and Sung JH: The pivotal role of reactive oxygen species generation in the hypoxia-induced stimulation of adipose-derived stem cells. Stem Cells Dev 20: 1753-1761, 2011.

26. Kim JH, Song SY, Park SG, Song SU, Xia Y and Sung JH: Primary involvement of NADPH oxidase 4 in hypoxia-induced generation of reactive oxygen species in adipose-derived stem cells. Stem Cells Dev 21: 2212-2221, 2012.

27. Park SG, Kim JH, Xia Y and Sung JH: Generation of reactive oxygen species in adipose-derived stem cells: Friend or foe? Expert Opin Ther Targets 15: 1297-1306, 2011.

28. Kim WS, Park BS, Park SH, Kim HK and Sung JH: Antiwrinkle effect of adipose-derived stem cell: Activation of dermal fibroblast by secretory factors. J Dermatol Sci 53: 96-102, 2009.
29. Kim WS, Park BS, Sung JH, Yang JM, Park SB, Kwak SJ and Park JS: Wound healing effect of adipose-derived stem cells: A critical role of secretory factors on human dermal fibroblasts. J Dermatol Sci 48: 15-24, 2007.

30. Jeong YM, Sung YK, Kim WK, Kim JH, Kwack MH, Yoon I, Kim DD and Sung JH: Ultraviolet B preconditioning enhances the hair growth-promoting effects of adipose-derived stem cells via generation of reactive oxygen species. Stem Cells Dev 22: 158-168, 2013.

31. Kue PF, Taub JS, Harrington LB, Polakiewicz RD, Ullrich A and Daaka Y: Lysophosphatidic acid-regulated mitogenic ERK signaling in androgen-insensitive prostate cancer PC-3 cells. Int J Cancer 102: 572-579, 2002

32. Hu X, Haney N, Kropp D, Kabore AF, Johnston JB and Gibson SB Lysophosphatidic acid (LPA) protects primary chronic lymphocytic leukemia cells from apoptosis through LPA receptor activation of the anti-apoptotic protein AKT/PKB. J Biol Chem 280: 9498-9508, 2005.

33. Yang JH, Jiang W, Pan CS, Qi YF, Wu QZ, Pang YZ and Tang CS: Effects of adrenomedullin on cell proliferation in rat adventitia induced by lysophosphatidic acid. Regul Pept 121: 49-56, 2004.

34. Boesch-Saadatmandi C, Wagner AE, Wolffram S and Rimbach G: Effect of quercetin on inflammatory gene expression in mice liver in vivo - role of redox factor 1, miRNA-122 and miRNA-125b. Pharmacol Res 65: 523-530, 2012.

35. Ishimoto T, Sugihara H, Watanabe M, Sawayama H, Iwatsuki M, Baba Y, Okabe H, Hidaka K, Yokoyama N, Miyake K, et al: Macrophage-derived reactive oxygen species suppress miR-328 targeting CD44 in cancer cells and promote redox adaptation. Carcinogenesis 35: 1003-1011, 2014.

36. Marin T, Gongol B, Chen Z, Woo B, Subramaniam S, Chien S and Shyy JY: Mechanosensitive microRNAs-role in endothelial responses to shear stress and redox state. Free Radic Biol Med 64: 61-68, 2013. 